\title{
Efecto de la densidad de siembra y la adición de ácido ascórbico en el cultivo de Osteoglossum bicirrhosum
}

\author{
Effect of stocking density and inclusion of ascorbic acid in \\ Osteoglossum bicirrhosum
}

\author{
Carolina Cuaical T, ${ }^{1 *}$ Ing, Evelyn Vallejo $\mathrm{V}^{1}{ }^{1} \mathrm{Ing}$, Hugo Franco R, ${ }^{2}$ M.Sc, \\ Wilmer Sanguino $0,{ }^{1}$ Ing.
}

\begin{abstract}
${ }^{1}$ Universidad de Nariño, Facultad de Ciencias Pecuarias, Departamento de Recursos Hidrobiológicos.

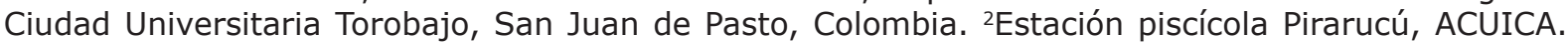
Florencia, Colombia.*Correspondencia: yenicarolina1@hotmail.com
\end{abstract}

Recibido: Junio de 2012; Aceptado: Marzo de 2013.

\section{RESUMEN}

Objetivo. Evaluar el efecto de la densidad de siembra y la adición de ácido ascórbico en la alimentación de alevinos de $O$. bicirrhosum, cultivados en jaulas. Materiales y métodos. Se incluyeron 396 alevinos y se distribuyeron en nueve tratamientos, resultado de la combinación de los niveles del factor densidad de siembra: 1pez/5 L; 1 pez/10 L y 1pez/15 L y los niveles de ácido ascórbico: 0; 500 y $1000 \mathrm{mg} / \mathrm{kg}$. Los datos obtenidos se evaluaron mediante el análisis de varianza del diseño factorial $3^{2}$. Resultados. La densidad de siembra presentó diferencias estadísticas significativas en el incremento de peso. Las variables incremento de longitud y tasa de crecimiento específico no presentaron diferencias estadísticas. En cuanto a la conversión alimenticia aparente, se demostraron diferencias significativas en los dos factores: La densidad de 1pez/10 L indicó la mejor conversión con un valor de 1.50, asimismo, la inclusión de $1000 \mathrm{mg}$ de ácido ascórbico por $\mathrm{Kg}$ de alimento mostró los mejores promedios con 1.55. En la sobrevivencia se precisaron diferencias estadísticas entre los tratamientos de la densidad de $1 \mathrm{pez} / 5 \mathrm{~L}$, siendo mejores aquellos que incluyeron ácido ascórbico en la dieta. Conclusiones. La densidad de $1 \mathrm{pez} / 10 \mathrm{~L}$ registró el mayor incremento de peso con 31.83 g en 87 días. La sobrevivencia general fue del $96 \%$, lo que demuestra la eficiencia del sistema de cultivo utilizado, al tiempo que actúa de manera positiva en el desarrollo de esta especie.

Palabras clave: Acuicultura, Cultivo, densidad de siembra, peces (Fuentes: AIMS, FAO). 


\section{ABSTRACT}

Objective. Evaluate the effect of planting density and inclusion of ascorbic acid in grown in cages fingerlings of $O$. bicirrhosum. Materials and methods. 396 fingerlings were studied and distributed in nine treatments, result of the combination of planting levels factor: 1 fish/ $5 \mathrm{~L} ; 1$ fish/10 L and 1 fish/15 $\mathrm{L}$ and ascorbic acid levels: 0,500 and $1000 \mathrm{mg} / \mathrm{Kg}$. Data were evaluated by variance analysis of $3^{2}$ factorial design. Results. The planting density showed statistically significant differences in weight gain. The length increase and specific growth rate variables were not statistically different. In terms of apparent feed conversion, it was shown significant differences in the two factors: 1 fish/10 L density indicated the best conversion with a value of 1.50; also, the inclusion of $1000 \mathrm{mg}$ ascorbic acid per $\mathrm{Kg}$ of feed showed the best average with 1.55. Referring to survival there was a statistical difference between treatments of 1 fish/ $5 \mathrm{~L}$ density, being the best those which included ascorbic acid in the diet. Conclusions. 1fish/10 L density had the largest increase of weight $31.83 \mathrm{~g}$ in 87 days. The general survival was $96 \%$, which, demonstrates the efficiency of the cropping system used, while it's acting positively on the development of this species.

Key words: Aquaculture, cultivation, fishes, stocking density (Sources: AIMS, FAO).

\section{INTRODUCCIÓN}

La Amazonia colombiana hace parte de la gran región con mayor biodiversidad en el planeta, posee importantes fuentes hídricas, las cuales brindan oportunidades sostenibles de producción y conservación de los recursos ícticos como prioridad frente al deterioro de sus hábitats naturales. La Arawana plateada originaria de la cuenca Amazónica y perteneciente a la familia Osteoglossidae, es considerada, al igual que los demás miembros de su familia, como pez con características antiguas, cuya particularidad es su lengua ósea (1).

Esta especie constituye uno de los recursos ícticos más promisorios, debido a su importancia ornamental, representado en el alto valor comercial con la generación de excelentes ingresos de divisas $(2,3)$. A partir del año 1970 adquiere una gran demanda en el mercado exterior, principalmente en los países asiáticos (4), donde influye en los factores culturales. La demanda a nivel internacional se concentra en dos etapas de su desarrollo, en un $93 \%$ en la fase de alevino: individuos menores de $10 \mathrm{~cm}$ y un $7 \%$ en los juveniles: individuos de 10 a $20 \mathrm{~cm}$ (5).

Sin embargo, la mayoría de los animales exportados corresponden a peces capturados del medio natural, generando desequilibrios en las poblaciones naturales y deterioro de los recursos hídricos (6). La sobre explotación de la especie y el manejo irracional de captura involucra generalmente el sacrificio de los machos incubantes para extraer de su boca las larvas; situación que le confiere un riesgo moderado de extinción o deterioro poblacional a mediano plazo, por lo que se le cataloga como una especie vulnerable (7); además, su condición de baja fecundidad (8) la hace más sensible ante la explotación pesquera.

Si bien existen metodologías básicas para el levante de esta especie en cautiverio, es necesario desarrollar estudios e incorporar nuevos elementos tecnológicos que permitan generar eficiencia en los sistemas productivos, mejoramiento y fortalecimiento en las técnicas de levante de juveniles de $O$. bicirrhosum optimizando su potencial productivo, contribuyendo a reducir su impacto ecológico y garantizando una producción sostenible, de tal forma que los altos precios actuales se mantengan gracias a certificaciones o sellos verdes en los cuales se evidencie el adecuado manejo y conservación de la especie (9). Por lo tanto, la presente investigación pretende aportar elementos en la optimización de manejo y cultivo, a través de dietas enriquecidas con ácido ascórbico y la evaluación del efecto de la densidad de siembra, de manera que garantice alta sobrevivencia, buen rendimiento en el crecimiento de los alevinos y asegure un uso racional, lícito, que potencialice los beneficios de la comercialización y competitividad, ayudando a preservar el equilibrio ecológico de la especie.

Teniendo en cuenta lo anterior, el objetivo de la investigación fue evaluar mediante un diseño factorial $3^{2}$ el efecto de la densidad de siembra e inclusión de ácido ascórbico en la alimentación de alevinos de $O$. bicirrhosum, cultivados en jaulas.

\section{MATERIALES Y MÉTODOS}

Sitio de estudio. El estudio se realizó en la Granja Santo Domingo de la Universidad de la 
Amazonia en convenio con la Piscícola Pirarucú, ubicada a $7 \mathrm{~km}$ de Florencia - Caquetá, entre las coordenadas geográficas $1^{\circ} 36^{\prime} 26^{\prime \prime}$ de latitud Norte y $75^{\circ} 31^{\prime} 58^{\prime \prime}$ de longitud Oeste, en la zona noroccidental del departamento. La Granja Santo Domingo se sitúa a una altitud promedio de $280 \mathrm{msnm}$, presenta temperatura ambiente promedio de $28^{\circ} \mathrm{C}$, humedad relativa entre 80 y $85 \%$, precipitación promedio anual de $3835 \mathrm{~mm}$ y brillo solar de 2000 horas (10).

Material biológico. Se estudiaron 396 alevinos de Arawana plateada, con un peso promedio de $16.73 \pm 7.15 \mathrm{~g}$, una longitud total promedio de $15.38 \pm 2.17 \mathrm{~cm}$ y una edad de 60 días aproximadamente. El análisis estadístico para peso y longitud inicial mostró homogenidad en todos los tratamientos. Los peces fueron obtenidos por reproducción natural en la misma estación y se adaptaron al consumo de dietas comerciales mediante un proceso de entrenamiento alimentario, cuya duración fue de 60 días.

Instalaciones. La adaptación al consumo del alimento comercial se realizó en el laboratorio de la estación piscícola, para este fin se utilizó una pileta rectangular con un volumen de $3552 \mathrm{~L}$ de capacidad. La etapa de ensayo se desarrolló en un estanque excavado en tierra de $4650 \mathrm{~m}^{2}$, al cual se le realizó labores previas de limpieza y desinfección e instalación de jaulas plásticas con capacidad de $120 \mathrm{~L}$, malla para la protección contra los depredadores y malla polisombra para disminuir los rayos directos del sol sobre los alevinos.

Manejo. Los peces se distribuyeron al azar. La combinación de los niveles del factor densidad de siembra: 1pez/5 L; 1 pez/10 L y 1 pez/15 L y los niveles de ácido ascórbico (L-ascorbyl-2monofosfato $35 \%(\mathrm{p} / \mathrm{v}))$ : $0 ; 500$ y $1000 \mathrm{mg}$ de ácido ascórbico por $\mathrm{Kg}$ de alimento comercial (45\% PB) conformaron los tratamientos (Tabla 1). Cada tratamiento se evaluó con tres replicas para un total de 27 unidades experimentales (Figura 1).

El suministro del alimento se realizó de acuerdo a la biomasa registrada en cada muestreo; en los dos

Tabla 1. Conformación de los Tratamientos.

\begin{tabular}{cccc}
\hline Tratamiento & Factor densidad & & $\begin{array}{c}\text { Factor ácido ascórbico } \\
(\mathbf{m g} / \mathbf{K g})\end{array}$ \\
\hline T1 & $1 \mathrm{PEZ} / 5 \mathrm{~L}$ & + & 0 \\
$\mathrm{~T} 2$ & $1 \mathrm{PEZ} / 10 \mathrm{~L}$ & + & 500 \\
$\mathrm{~T} 3$ & $1 \mathrm{PEZ} / 15 \mathrm{~L}$ & + & 1000 \\
$\mathrm{~T} 4$ & $1 \mathrm{PEZ} / 5 \mathrm{~L}$ & + & 500 \\
$\mathrm{~T} 5$ & $1 \mathrm{PEZ} / 10 \mathrm{~L}$ & + & 1000 \\
T6 & $1 \mathrm{PEZ} / 15 \mathrm{~L}$ & + & 0 \\
$\mathrm{~T} 7$ & $1 \mathrm{PEZ} / 5 \mathrm{~L}$ & + & 1000 \\
T8 & $1 \mathrm{PEZ} / 10 \mathrm{~L}$ & + & 0 \\
T9 & $1 \mathrm{PEZ} / 15 \mathrm{~L}$ & + & 500 \\
\hline
\end{tabular}

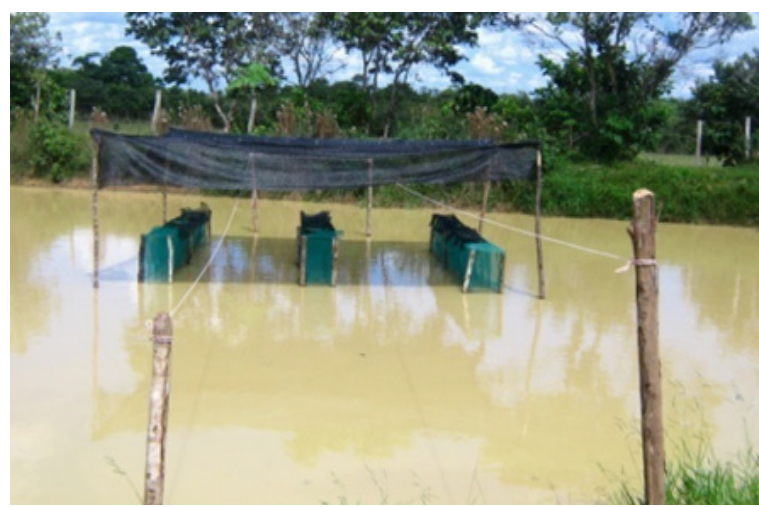

Figura 1. Unidades experimentales.

primeros meses se proporcionó alimento al $2.4 \%$ de la biomasa y en el último mes se ajustó al 2.2\% de la biomasa de cada tratamiento, la ración diaria se dividió en cuatro comidas.

Para determinar el desempeño productivo a lo largo de la investigación, se realizaron muestreos quincenales, tomando el $50 \%$ de la población de cada tratamiento, para esto se registró el peso $(\mathrm{g})$ y la longitud total $(\mathrm{cm})$. Al finalizar el período de estudio, se realizó un censo total de la población.

Los parámetros físicos y químicos del agua: $\mathrm{pH}$, temperatura, oxígeno disuelto, alcalinidad, dureza y amonio se determinaron mediante un kit de análisis de agua tipo HACH modelo FF-1 Cat No. 2430-02.

Diseño experimental y análisis estadístico. Las variables evaluadas fueron:

Ganancia de peso [GP=(peso final (g)-peso inicial $(\mathrm{g})]$, Ganancia de talla $[\mathrm{GT}=$ (talla final $(\mathrm{cm})$ - talla inicial $(\mathrm{cm})]$,

Tasa de crecimiento específico $[\mathrm{TCE}=((\mathrm{In}$ peso final - In peso inicial)/ tiempo de estudio)*100],

Factor de conversión alimenticia aparente $[C A A=$ (alimento suministrado $(\mathrm{g}) /$ ganancia de peso(g)] y la

Tasa de sobrevivencia $[\mathrm{TS}=$ (No final de peces/ No inicial de peces)*100].

Las variables se estudiaron mediante el análisis de varianza del diseño factorial $3^{2}$, en la variable tasa de crecimiento específico se aplicó la prueba no paramétrica de Kruskal Wallis; en aquellas que existieron diferencias estadísticas significativas se utilizó la prueba de comparaciones múltiples de Tukey. Para el caso de la variable sobrevivencia se empleó la prueba de Brand Snedecor. El nivel de significancia estadístico fue $\mathrm{p}<0.05$. 


\section{RESULTADOS}

Los valores promedio de los parámetros físicos y químicos del agua se muestran en la tabla 2.

Tabla 2. Promedios de parámetros físicos y químicos del agua

\begin{tabular}{lc}
\hline \multicolumn{1}{c}{ Parámetros } & Valores \\
\hline Alcalinidad $\left(\mathrm{mg} / \mathrm{L} \mathrm{CaCO}_{3}\right)$ & $34.2 \pm 6.2$ \\
Dureza $\left(\mathrm{mg} / \mathrm{L} \mathrm{CaCO}_{3}\right)$ & $20.9 \pm 9.4$ \\
Temperatura $\left({ }^{\circ} \mathrm{C}\right)$ & $26.9 \pm 0.9$ \\
$\mathrm{pH}$ & $6.6 \pm 0.2$ \\
Oxígeno $(\mathrm{mg} / \mathrm{L})$ & $4.8 \pm 0.1$ \\
Amonio $(\mathrm{mg} / \mathrm{L})$ & $0.5 \pm 0.09$ \\
\hline
\end{tabular}

En periodos de lluvias intensas la temperatura no presentó oscilaciones drásticas puesto que el área del estanque permitió mantener mayor estabilidad de los parámetros de calidad del agua. De la misma manera, en los días muy soleados se presentó un aumento y descenso gradual de este parámetro en el transcurso del día, alcanzando valores máximos de $32^{\circ} \mathrm{C}$, siendo la variación de forma gradual y, no se observaron efectos negativos en los juveniles de Arawana.

Con relación a las variables productivas, la densidad de siembra presentó un efecto estadísticamente significativo en la ganancia de peso de los juveniles de Arawana plateada, estableciéndose los mejores promedios en las densidades de $1 \mathrm{pez} / 10 \mathrm{~L}$ y 1 pez/15 L con 31.83 g y 29.83 g respectivamente (Figura 2).

La figura 2 relaciona los promedios de ganancia de peso alcanzados en cada nivel de densidad de siembra, en la figura, la barra que representa la densidad $1 \mathrm{pez} / 10 \mathrm{~L}$ no se intersecta con la barra de la densidad 1pez/5 L, por lo tanto son diferentes estadísticamente.

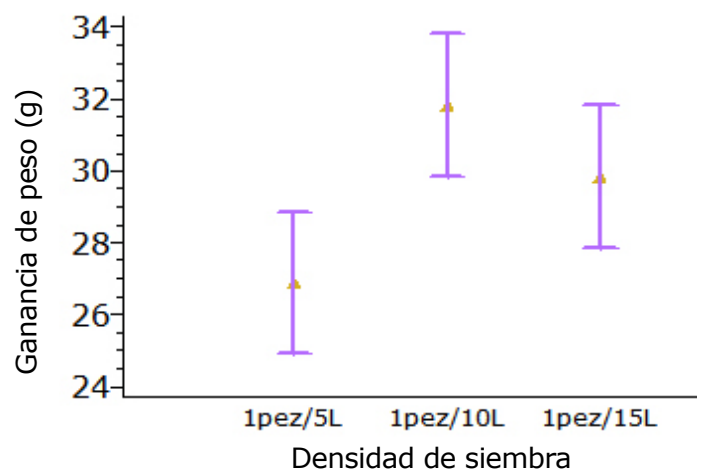

Figura 2. Promedios de ganancia de peso para cada nivel de densidad de siembra.
Respecto a la inclusión de ácido ascórbico en el alimento y la interacción de los dos factores de estudio no se precisaron diferencias estadísticas significativas en la ganancia de peso; sin embargo, los valores de ganancia de peso fueron menores en los tratamientos que no recibieron ácido ascórbico con respecto a los grupos con dietas suplementadas. Los valores promedios en cada nivel fueron: $27.59 \mathrm{~g} ; 30.24 \mathrm{~g}$ y $30.72 \mathrm{~g}$ para $0 \mathrm{mg} / \mathrm{kg} ; 500 \mathrm{mg} / \mathrm{kg}$ y $1000 \mathrm{mg} / \mathrm{kg}$ respectivamente.

En la variable ganancia de longitud, los factores de estudio y la interacción de estos no presentaron efecto estadístico significativo, los valores promedio para el factor vitamina C fueron: $5.8 \mathrm{~cm}(0 \mathrm{mg} / \mathrm{kg})$, $6.2 \mathrm{~cm}(1000 \mathrm{mg} / \mathrm{kg})$ y $6.4 \mathrm{~cm}(500 \mathrm{mg} / \mathrm{kg})$; en el factor densidad de siembra los valores promedios conseguidos corresponden a: 1pez/5 L: $5.8 \mathrm{~cm}$; $1 \mathrm{pez} / 10 \mathrm{~L}: 6.6 \mathrm{~cm}$ y $1 \mathrm{pez} / 15 \mathrm{~L}: 6 \mathrm{~cm}$.

Referente al factor de conversión alimenticia aparente (CAA), la inclusión de ácido ascórbico y la densidad de siembra presentaron un efecto estadístico significativo para esta variable. Además, la prueba de contraste múltiple de Tukey, determinó diferencias significativas en los tratamientos sin adición de vitamina con los tratamientos de $1000 \mathrm{mg} / \mathrm{kg}$; de este modo, los promedios de 500 y $1000 \mathrm{mg} / \mathrm{kg}$ fueron estadísticamente iguales e indicaron los mejores resultados de conversión alimenticia con 1.55 para 1000 mg/ kg y 1.60 para 500 mg/kg (Figura 3).

En la figura 3 el nivel de $1000 \mathrm{mg} / \mathrm{kg}$ es diferente estadísticamente con el nivel $0 \mathrm{mg} / \mathrm{Kg}$, las barras de estos dos niveles no se intersectan.

En los niveles de densidad de siembra, los resultados mostraron diferencias significativas para la conversión alimenticia entre los promedios de los tratamientos de $1 \mathrm{pez} / 5 \mathrm{~L}$ y $1 \mathrm{pez} / 10 \mathrm{~L}$.

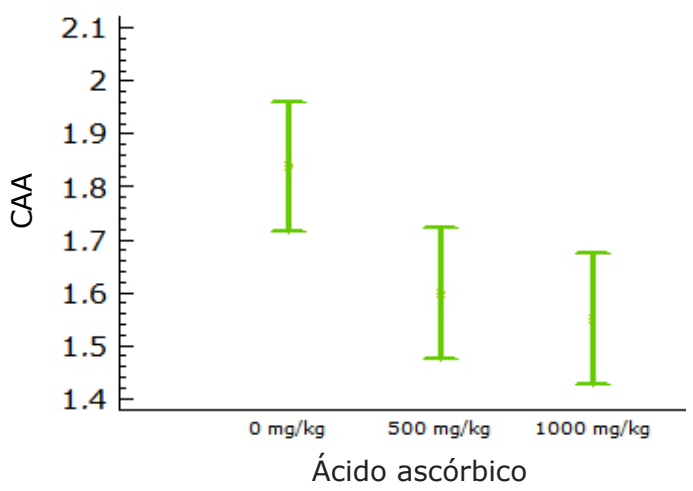

Figura 3. Promedios de conversión alimenticia (CAA) para cada nivel de ácido ascórbico. 
También se estableció la mejor conversión en la densidad de $1 \mathrm{pez} / 10 \mathrm{~L}$ con un valor de 1.50 (Figura 4).

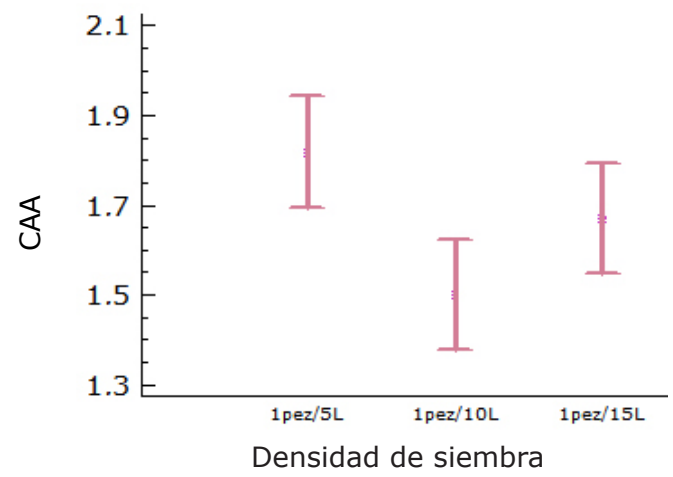

Figura 4. Promedios de conversión alimenticia para cada nivel de densidad de siembra.

La figura 4 indica diferencias significativas entre los niveles de 1 pez/5 L y $1 \mathrm{pez} / 10 \mathrm{~L}$, en la figura, las barras de los niveles no se intersectan.

En cuanto a la tasa de crecimiento específico, no hubo diferencias estadísticas significativas en los factores de estudio ni la interacción entre ellos. Sin embargo, los promedios de los tratamientos con inclusión de $1000 \mathrm{mg}$ de ácido ascórbico por $\mathrm{Kg}$ de alimento consiguieron un mayor porcentaje de crecimiento diario. Igualmente, los promedios de la densidad de 1pez/10 L alcanzaron los mejores resultados. Los valores promedios se indican en la tabla 3.

Tabla 3. Promedios de tasa de crecimiento específica

\begin{tabular}{cccccc}
\hline Niveles de ácido ascórbico & \multicolumn{4}{c}{$\begin{array}{c}\text { Niveles de densidad de } \\
\text { siembra }\end{array}$} \\
\hline $0 \mathrm{mg} / \mathrm{Kg}$ & $500 \mathrm{mg} / \mathrm{Kg}$ & $1000 \mathrm{mg} / \mathrm{Kg}$ & $1 \mathrm{pez} / 5 \mathrm{~L}$ & $1 \mathrm{pez} / 10 \mathrm{~L}$ & $1 \mathrm{pez} / 15 \mathrm{~L}$ \\
$1.12 \%$ & $1.19 \%$ & $1.26 \%$ & $1.09 \%$ & $1.26 \%$ & $1.22 \%$ \\
\hline
\end{tabular}

Respecto a la variable sobrevivencia, se encontró diferencias significativas entre los tratamientos T1, T4 y T7. En los demás tratamientos no se presentaron diferencias estadísticas.

\section{DISCUSIÓN}

El incremento de peso y longitud por muestreo presentaron una conducta variable, en la que se observaron aumentos y descensos. Este tipo de comportamiento en el crecimiento puede estar asociado con la cantidad de alimento ingerido. Pues, en días de lluvias intensas, no se suministró la cantidad total de alimento calculado para cada ración, debido a que los peces reducen su metabolismo y se pierde el alimento no consumido; en los juveniles de Arawana se notó ocultamiento e inapetencia por el alimento $y$, en efecto concuerda con los incrementos más bajos conseguidos en esos períodos. A pesar que la fluctuación de los parámetros físicos y químicos del agua no fue drástica, se observó que el promedio más bajo de temperatura también coincide con valores promedios menores de crecimiento. El crecimiento de los peces está ligado a un factor de origen biológico de tal modo que, cualquier factor del ambiente interactúa con él. En condiciones naturales si la temperatura aumenta, la cantidad de alimento ingerido normalmente aumenta, así como la tasa de digestión. La tasa de crecimiento puede aumentar o disminuir dependiendo de la relación entre alimento-metabolismo-temperatura (11).

Además, el reporte de valores bajos para dureza, clasifica el agua de tipo blanda, lo cual es característico de la región Amazónica, por otra parte, la alcalinidad y dureza se mantuvieron en valores similares, demostrando condiciones adecuadas de calidad.

Los promedios de ganancia de peso y longitud alcanzados en cada nivel de densidad de siembra, indicaron que a mayor densidad de cultivo se consigue menor ganancia de peso y longitud. Sin embargo, al comparar la ganancia de peso promedio para la densidad media y baja, se observó en la densidad media (1pez/ $10 \mathrm{~L}$ ) mejores incrementos, probablemente los aspectos relacionados con el comportamiento gregario de la especie puede influir en su desarrollo; dado que esta conducta la hace muy sociable y dócil (7). De la misma manera, en los tratamientos con densidades de 1pez/15 L se notó menos agilidad al consumir alimento: la cantidad de alimento suministrado fue menor con respecto a la densidad de 1 pez/10 L; de tal manera que, la mayor disponibilidad de alimento motivaba a los juveniles de Arawana a salir rápidamente a consumir. Tendencias semejantes en relación al consumo de alimento se observaron en juveniles de Arapaima gigas al evaluar el efecto de la densidad de siembra sobre la eficiencia alimenticia, los resultados indicaron que el incremento de la densidad de siembra mejora la conversión alimenticia, el consumo de alimento, la condición física de los peces y reduce los costos (12).

En la variable ganancia de longitud, los factores de estudio y la interacción de estos no presentaron efecto estadístico significativo; no obstante, hubo mejor desarrollo productivo en los tratamientos 
con suplementación de ácido ascórbico en la dieta y en aquellos que manejaron una densidad de 1 pez/10 L con lo cual se favorece el sistema productivo al tener en cuenta que en estos tratamientos la sobrevivencia fue mayor. Ya que el principal objetivo en la producción de peces ornamentales está condicionado con el número final de individuos y, que en general los avances investigativos de los sistemas productivos tienen como principio generar modelos eficientes de producción que garantice una buena rentabilidad.

De otra parte, los resultados del factor de conversión alimenticia indicaron mejores valores en los tratamientos con inclusión de ácido ascórbico en la dieta y a la vez son los que mejor ganancia de peso obtuvieron, demostrando que la vitamina $\mathrm{C}$ mejoró la utilización de los nutrientes y por consiguiente sus índices productivos.

Además, es importante resaltar que los peces no pueden sintetizar ácido ascórbico debido a que carecen de actividad de la enzima responsable de la síntesis a partir de la glucosa, consecuentemente es necesario suministrar en la dieta para cubrir sus requerimientos nutricionales. El ácido ascórbico está involucrado en varias funciones fisiológicas gracias a su buen agente redactor en las que incluye el crecimiento, desarrollo, reproducción, cicatrización, respuesta al estrés entre otros procesos (13); igualmente, esta vitamina desempeña un papel importante en la formación de colágeno, que es el principal componente del esqueleto $y$, por lo tanto necesario para el desarrollo del organismo por lo que actúa directamente en el crecimiento de los peces (14). De este modo, se sugiere incorporar en las dietas artificiales para peces ácido ascórbico, con el propósito, no sólo de cubrir las necesidades fisiológicas, sino también estimular el sistema inmunológico y reducir la vulnerabilidad al ataque de diferentes agentes etiológicos (15).

En investigaciones realizadas con inclusión de ácido ascórbico, se reportó mejor crecimiento y eficiencia alimenticia en alevinos de carpa india (Labeo rohita) alimentados con dietas suplementadas con ácido ascórbico (16). También, un estudio en Paralichthys olivaceus determinaron resultados de ganancias de peso y tasa de eficiencia proteica significativamente menores en el tratamiento sin inclusión de ácido ascórbico de aquellos con niveles de inclusión; además, observaron signos de deficiencia de dicha vitamina, tales como anorexia, escoliosis, cataratas y hemorragias en las aletas (17). Otra investigación realizada en alevinos de Heterobranchus longifilis se notó signos de deficiencia cuyo tratamiento no se adicionó vitamina, caracterizadas por lordosis, deformidad de la aleta caudal y erosión en la piel, al tiempo que la ganancia de peso fue significativamente menor (18).

Los resultados de sobrevivencia indicaron menor porcentaje de sobrevivencia en los tratamientos T1 y T6 cuyas dietas no se incluyó vitamina C, los valores fueron: $88.89 \%$ y $87.50 \%$ respectivamente; para los tratamientos T3, T4, T7 los resultados de sobrevivencia correspondieron en su orden: $91.7 \% ; 97.2 \%$ y $98.6 \%$. En los tratamientos T2, T5, T8 y T9 se registraron sobrevivencias del $100 \%$, demostrando que la densidad de $1 \mathrm{pez} / 10 \mathrm{~L}$ es adecuada y permite obtener una buena producción bajo las condiciones del estudio. En términos generales, se obtuvo una sobrevivencia total del $96 \%$, lo que indica la eficiencia del sistema de cultivo utilizado, sugiriendo que las diferentes densidades de siembra y niveles de ácido ascórbico adicionado en el alimento actúan de manera positiva en el desarrollo de esta especie cultivada en jaulas.

Los resultados de un estudio realizado en juveniles de Pterophylum scalare alimentados con artemia enriquecida con la combinación de a-tocoferol y vitamina $\mathrm{C}$ mostraron mejores tasas de sobrevivencia, mayor ganancia de peso y aumento de la resistencia al estrés osmótico (19). De igual forma, se consiguió mayor sobrevivencia y mejor crecimiento en larvas de carpa alimentadas con zooplancton enriquecido con ácido ascórbico que en aquellos que no se enriqueció y, se concluyó que este método garantiza la calidad en el cultivo de larvas a un costo y tiempo mínimo (20).

Cabe destacar que el uso de ácido ascórbico en la dieta de los ejemplares actuó como inmunoestimulante disminuyendo el estrés ocasionado durante el ciclo productivo, por lo que la sobrevivencia fue mucho más alta. Corredor y Landines (21) indican la necesidad de implementar estrategias que contribuyan a disminuir el impacto de los diferentes factores estresantes a los que son sometidos los peces durante su proceso de producción en cautiverio. En virtud de eso, la vitamina C es la más utilizada para mejorar la resistencia a enfermedades, por lo que es un componente esencial para el normal funcionamiento de los peces, contribuye en el crecimiento de los animales y aumenta su capacidad de resistencia frente a enfermedades, condiciones adversas y de estrés.

Varios estudios desarrollados en peces y camarones con dietas suplementadas con ácido ascórbico y en los que se ha aplicado pruebas 
de estrés como: salinidad, exposición aérea, estrés por baja o alta temperatura en el agua, exposición a agentes patógenos, sustancias tóxicas y heridas causadas en la piel se ha demostrado mejor respuesta inmune, debido al aumento de la capacidad de respuesta fisiológica al estrés.

De este modo, al enriquecer la dieta con niveles de $500,800,1200 \mathrm{mg}$ de vitamina C y E por $\mathrm{kg}$ de alimento sobre las respuestas fisiológicas de Arapaima gigas, se determinó un efecto significativo en los niveles altos de vitamina $C$ (22); de la misma manera, se concluyó que la suplementación dietética con ácido ascórbico juega un papel importante en la mitigación de los efectos negativos del estrés crónico en los juveniles de Leiocassis longirostris expuestos a amoniaco (23). En Oreochromis niloticus sometidos a exposición de plomo, observaron la eficiencia del ácido ascórbico en la reducción de la genotoxicidad por este elemento químico (24). Igualmente, se señaló que el requerimiento de ácido ascórbico en la dieta para Notemigonus crysoleucas incrementa la respuesta al estrés causado por la exposición a alta temperatura del agua (25).

En conclusión, la densidad de siembra mostró un efecto estadísticamente significativo en la ganancia de peso de los juveniles de Osteoglussum bicirrhosum cultivados en jaulas; indicando los mejores promedios en las densidades de $1 \mathrm{pez} / 10 \mathrm{~L}$ y $1 \mathrm{pez} / 15 \mathrm{~L}$, con valores de 31.83 y $29.83 \mathrm{~g}$ respectivamente. La sobrevivencia general del $96 \%$ demuestra la eficiencia del sistema de cultivo utilizado y determina que las diferentes densidades de siembra y niveles de ácido ascórbico adicionado en el alimento actúan de manera positiva en el desarrollo de esta especie, comprobando al mismo tiempo la eficiencia de esta vitamina en la cicatrización rápida de las heridas. El cultivo intensivo de $O$. bicirrhosum en jaulas bajo esta tecnología de manejo mejora: la sobrevivencia, el crecimiento, la conversión alimenticia y disminuye la incidencia de enfermedades. Además, el costo no es elevado y es una opción adecuada que permite el aprovechamiento racional de esta importante especie íctica nativa, en una excelente y atractiva alternativa para la piscicultura de la Amazonía Colombiana.

\section{Agradecimentos}

A la estación Piscícola Pirarucú en convenio con la Universidad de la Amazonia, Caquetá, por su valioso apoyo logístico y financiero, al programa de Ingeniería en Producción Acuícola de la Universidad de Nariño y a todas las personas que de alguna u otra manera colaboraron en el desarrollo de esta investigación.

\section{REFERENCIAS}

1. Santos G, Ferreira E, Zuanon J. Peixes comerciais de Manaus. Manaus: ProVárzea, 2006.

2. Mojica JI, Usma Oviedo JS, Álvarez León R, Lasso C, editores. Libro rojo de peces dulceacuícolas de Colombia. Bogotá, Colombia: Instituto de Investigación de Recursos Biológicos Alexander von Humboldt, Instituto de Ciencias Naturales de la Universidad Nacional de Colombia, WWF Colombia, Universidad de Manizales; 2012.

3. Moreau M, Coomes O. Potential threat of the international aquarium fish trade to silver arawana Osteoglossum bicirrhosum in the Peruvian Amazon. Oryx 2006; 40(2):152-160.

4. Escobar MD, Farias IP, Taphorn DC, Landines M, Hrbek T. Molecular diagnosis of the arowanas Osteoglossum ferreirai Kanazawa, 1966 and $O$. bicirrhossum (Cuvier, 1829) from the Orinoco and Amazon River basins. Neotrop Ichthyol 2013; 11(2):335-340.
5. Agudelo-Zamora HD, López-Macías JN, Sánchez-Páez CL. Hábitos alimentarios de la Arawana (Osteoglossum bicirrhosum Vandelli, 1829) (Pisces: Osteoglossidae) en el alto río Putumayo, área del Parque nacional natural la Paya. Acta Biol Par (Curitiba) 2007; 36(1-2):91-101.

6. Mancera N, Álvarez L. Comercio de peces ornamentales en Colombia. Acta Biol Colomb 2008; 13(1):23-52.

7. Mojica JI, Castellanos Castillo C, Usma Oviedo JS, Álvarez León R, editores. Libro rojo de peces dulceacuícolas de Colombia. Serie Libros Rojos de Especies Amenazadas de Colombia. Bogotá, Colombia: Instituto de Ciencias NaturalesUniversidad Nacional de Colombia, Instituto Humboldt de Colombia, Ministerio del Medio Ambiente; 2002. 
8. Ortiz N, Iannacone J. Estado actual de los peces ornamentales amazónicos del Perú que presentan mayor demanda de exportación. Biologist 2008; 6(1):54-67.

9. Zuñiga-Upegui PT. Lineamientos de gestión ambiental para el control del tráfico ilícito de peces ornamentales dulceacuícolas de las cuencas Amazonas y Orinoco. Bogotá, Colombia: Pontificia Universidad Javeriana; 2010.

10. Corporación Colombiana de Investigación Agropecuaria CORPOICA. Estación metereológica Macagual. Florencia Caquetá Colombia: CORPOICA; 2005.

11. Anguas-Velez BH, Civera R, Goytortúa E, Rocha-Meza S. Efecto de la temperatura y la densidad de cultivo sobre el crecimiento de juveniles de la Cabrilla arenera, Paralabrax maculatofasciatus. Hidrobiológica 2003; 13(4):309-315.

12. Cavero BAS, Pereira-Filho M, Roubach $R$, Rabello-Ituassú D, Lima-Gandra A, Crescêncio R. Efeito da densidade de stocagem sobre a eficiencia alimentar de juvenis de Pirarucu (Arapaima gigas) em ambiente confinado. Acta Amaz 2003; 33(4):631-636.

13. Prieto M, Atencio V. Zooplancton en la larvicultura de peces neotropicales. Rev MVZ Córdoba 2008; 13(2):1415-1425.

14. Lima AF. Crescimiento de pós-larvas de tilapia do Nilo em função da densidade e da suplementação de vitamina $C$ na ração [Tesis de Maestría]. Recife, Brasil: Universidade Federal Rural de Pernambuco, Departamento de Pesca e Aqüicultura; 2010.

15. Guillaume J, Kaushik S, Bergot P, Metailler R. Nutrición y alimentación de peces y crustáceos. Madrid, España: Mundi-Prensa; 2004.

16. Misra CK, Das BK, Mukherjee SC, Pradhan J. Effects of dietary vitamin $C$ on immunity, growth and survival of Indian major carp Labeo rohita, fingerlings. Aquac Nutr 2007; 13:35-44.

17. Wang X, Kim K, Bai S. Effects of different dietary levels of L-ascorbyl 2- polyphosphate on growth and tissue vitamin $\mathrm{C}$ concentrations in juvenile olive flounder, Paralichthys olivaceus (Temminck et Schlegel). Aquac Res 2002; 33(4):231-309.
18. Ibiyo LMO, Atteh JO, Omotosho JS, Madu CT. Vitamin C (ascorbic acid) requirements of Heterobranchus longifilis fingerlings. Afr J Biotechnol 2007; 6(13):1559-1567.

19. Norouzitallab P, Farhangi M, Babapour M, Rahimi R, Sinha AK, Baruah K. Comparing the efficacy of dietary a-tocopherol with that of DL-a-tocopheryl acetate, both either alone or in combination with ascorbic acid, on growth and stress resistance of angelfish, Pterophylum scalare, juveniles. Aquac Int 2009; 17:207-216.

20. Mitra G, Mukhopadhyay PK. Dietary essentiality of ascorbic acid in rohu larvae: Quantification with ascorbic acid enriched zooplankton. Aquac Int 2003; 11:81-93.

21. Corredor AS, Landines MA. Efecto del ácido ascórbico sobre la respuesta de los peces ante condiciones de estrés. Rev Med Vet Zoot 2009; 56:53-66.

22. de Andrade JI, Ono EA, Cruz G, Martins-Brasil E, Roubach R, Criscuolo-Urbinati E, et al. Influence of diets supplemented with vitamins $\mathrm{C}$ and $\mathrm{E}$ on pirarucu (Arapaima gigas) blood parameters. Comp Biochem Physiol A Mol Integr Physiol 2007; 146(4):576-580.

23. Liu H, Xie S, Zhu X, Lei W, Han D, Yang Y. Effects of dietary ascorbic acid supplementation on the growth performance, immune and stress response in juvenile Leiocassis longirostris Günther exposed to ammonia. Aquac Res 2008:1-11.

24. Jiraungkoorskul W, Sahaphong S, Kangwanrangsan N, Zakaria S. The protective influence of ascorbic acid against the genotoxicity of waterborne lead exposure in Nile tilapia Oreochromis niloticus (L.). J Fish Biol 2008; 73:355-366.

25. Chen R, Lochmann R, Goodwin A, Praveen K, Dabrowski K, Lee KJ. Alternative complement activity and resistance to heat stress in Golden Shiners (Notemigonus crysoleucas) are increased by dietary Vitamin $C$ levels in excess of requirements for prevention of deficiency signs. J Nutr 2003; 133:2281-2286. 